\title{
Prediction of electronic nanodevices technical status and reliability based on analysis of their performance parameters kinetics under the influence of external factors
}

\author{
Mstislav Makeev ${ }^{1, *}$, Sergey Meshkov ${ }^{1}$, and Vladimir Sinyakin ${ }^{1}$ \\ ${ }^{1}$ Bauman Moscow State Technical University, 105005 Moscow, Russia
}

\begin{abstract}
The problems of technical diagnostics of electronic nanoobjects and devices based on them are considered. Algorithms for constructing the operability and reliability prediction fields of electronic nanoobjects and devices based on them in the specified operation conditions are given.
\end{abstract}

\section{Introduction}

One of the promising areas for the space industry development is the creation of a small spacecraft system, which will be entrusted with the solution of a wide range of tasks, both purely commercial and related to national security and the competitiveness of the country. Nanodevices that make it possible to give the equipment new qualitative characteristics and to increase the efficiency of performing the functions assigned to them are used for spacecraft hardware creation. The priority task of such nanodevices creation is the use of domestic materials, technologies and element base.

Strict requirements are imposed to the space-based nanodevice reliability. They are dictated by hard operating conditions on the one hand and the increasing spacecraft active shelf life on the other. The most reliable and complete reliability index of devices are usually obtained from the operation results. However, obtaining such information takes a lot of time. Traditional test methods in many cases also do not allow to confirm a given level of object reliability due to the presence of hidden defects. One of the promising directions in the development of methods for assessing the reliability and quality of nanodevices for space purposes is to predict their future status.

\section{Problem formulation}

The problem of the technical system reliability increasing with reliability prediction development on specified exploitation period in specified conditions is solved by technical diagnostics (TD), the scientific base of which are outlined particularly in $[1,2]$. One of the main problems of TD is the identification of the object status under conditions of limited information. Object status identification is based on identification algorithms, which are usually considered as classification problems.
Identification algorithms in technical diagnostics are based on the diagnostic models, which establish relation between object status and its representation in the diagnostic signals dimension. An important part of identification problem is decision-making rules. Enumerated elements of the TD structure need to be developed regarding to the specific object of diagnostics.

The purpose of this project is the development of the major elements of TD structure regarding to electronic nanocomponents, which characteristics determine the indices of devices based on it.

An example of such promising electronic nanoscale components are resonant tunneling diodes (RTDs) based on multilayered nanoscale $\mathrm{AlGaAs}$ nanoheterostructures. RTD operation principle is based on the physical effect of resonance tunneling of electrons in the nanocomponent heterostructure, which layer thickness is in the range from units to dozens of nanometers. The prospects of RTD for modern radio electronics is caused by the following circumstances:

- RTD operates in the range of temperatures and other external factors necessary for technical applications.

- Technologies and facilities for RTD production are validated in the framework of micro- and nanoelectronics technologies.

- The limit frequency for RTD spreads up to units of THz [3-6], which makes the RTD a promising device for SHF, EHF, and THF electronics.

- It is possible to control the shape of the current-voltage characteristic (CVC) and create a diode with the optimum CVC shape for the specific type of nonlinear conversion by changing the parameters of the heterostructure layers (thickness, chemical composition).

It was shown in [7-10] that the possibility of optimizing the CVC shape, as well as the presence of a falling part on it, makes it possible to create a number of nonlinear radio signals converters with improved characteristics based on RTD. The set of possible

\footnotetext{
*Corresponding author: $\underline{\text { mc.stiv@gmail.com }}$
} 
nonlinear transformations using RTD is very wide: generation of radio signals, frequency modulation, mixing of radio signals, amplitude detection, rectification, frequency labels grid generation and etc.

The following tasks are solved in the work with regard to electronic nanodevices:

- development of decision-making rules. The rules are meant for decision making on finding the parameters of a nanodevices in the field of operability.

- development of a methodology for constructing a reliability prediction of the nanocomponents and a devices based on it.

Herewith, two hypotheses are accepted:

- Reliability of nanocomponent and device based on it are based on gradual failure model [11].

- Degradation of device parameters is determined by nanocomponent degradation. Nanocomponent degradation leads to the changes of its CVC shape, resulting in device parameters (indices) changes. The failure of device and nanocomponent occurs, when at least one of the device indices falls outside of set limits range (operability field).

\section{Decision-making rules}

The rules of decision-making are meant for decision making of nanocomponent parameter location in the operability field. Fig. 1 shows in simplified form fields of operability $\boldsymbol{O}$, correct operation $\boldsymbol{C} \boldsymbol{O}$ and catastrophic failure $\boldsymbol{C F}$ in the context of RTD for radio signals mixers.

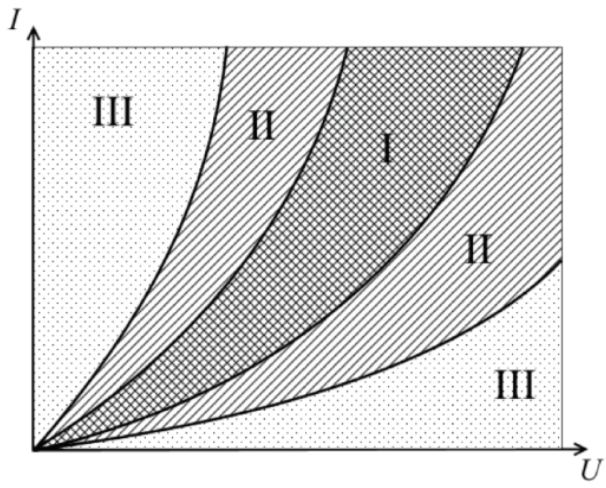

I - operability field $\boldsymbol{O}$, II - correct operation field $\boldsymbol{C} \boldsymbol{O}$, III - catastrophic failure $\boldsymbol{C F}$ field

Fig. 1. Fields of nanocomponent operability $\boldsymbol{O}$, correct operation $\boldsymbol{C O}$ and catastrophic failure $\boldsymbol{C F}$ in the context of RTD for radio signals mixers.

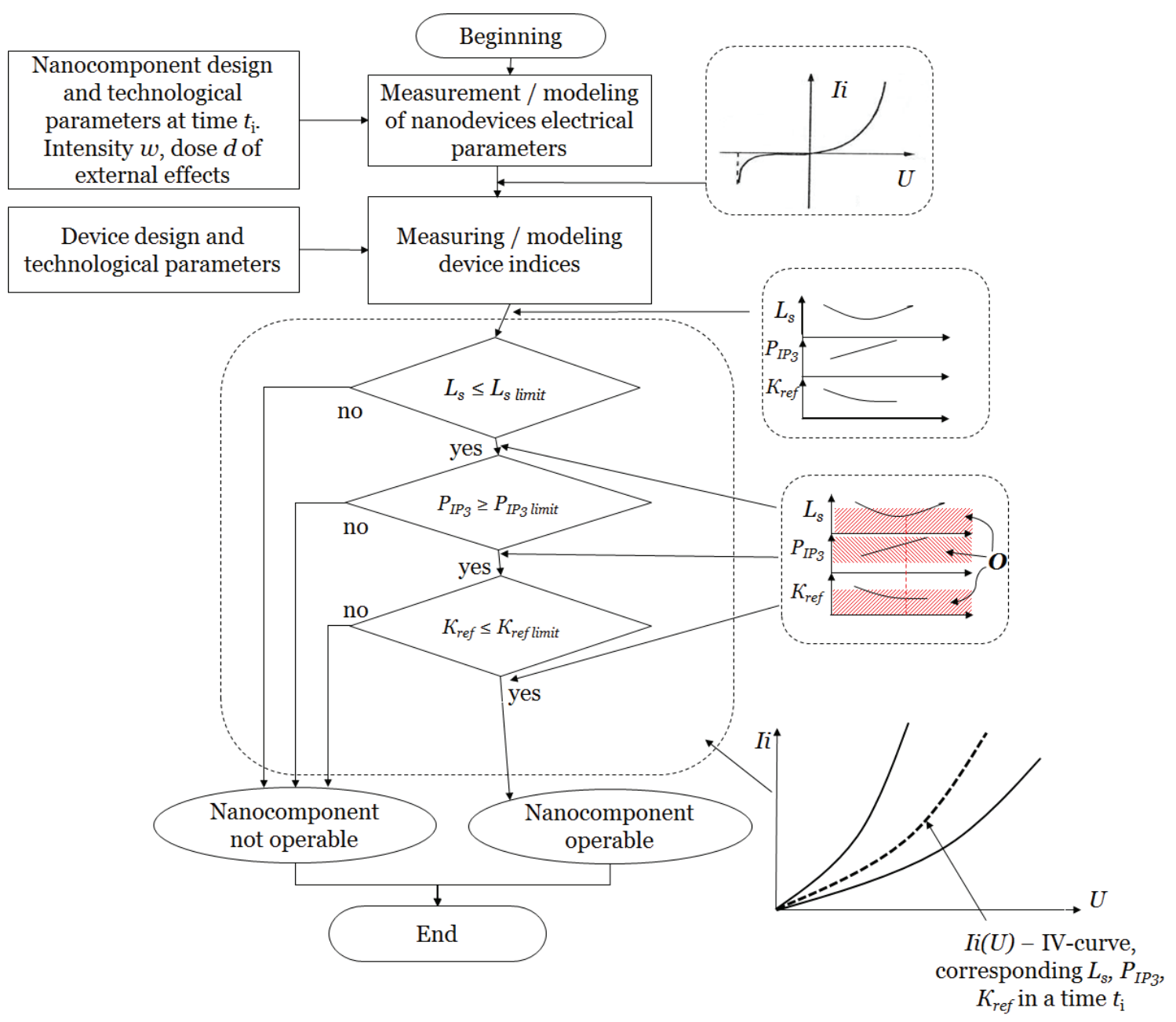

Fig. 2. Decision-making algorithm. 
The assignment of nanocomponent parameters to a particular category depends on the characteristics of the device based on it.

Decision-making algorithm of relating nanocomponent parameters to a certain category is shown in Fig.2. If we consider a radio signals mixer as the device the indices of which is determined by a nanocomponent, then we consider the conversion loss $L_{\mathrm{s}}$, the dynamic range $P_{\mathrm{IP} 3}$ and the input signal reflection coefficient $K_{\text {ref }}$ as the indices. We will assume that the mixer operability field is determined by the conditions:

$$
\begin{aligned}
& L_{s} \leq L_{\text {s limit }}, \\
& P_{I P 3} \geq P_{I P 3 \text { limit }}, \\
& K_{\text {ref }} \leq K_{\text {ref limit }} .
\end{aligned}
$$

Special feature of decision-making of the nanocomponent parameters classification involves operability field $\boldsymbol{O}$ transformation from device indices space into nanocomponent parameters plane, so that one point in device indices space $\left\{L_{\mathrm{si}}, P_{\mathrm{IP} 3 i}, K_{\text {refi }}\right\}$ corresponds to one curve $I_{i}\left(U_{i}\right)$.

At the first stage, the nanocomponent electrical parameters, which are transferred to a software module that calculates the device's indices, are measured or calculated. The result of the device simulation is its indices (1)-(3). In general, the program module responsible for calculating the device indices can be replaced by a measuring module, but this replacement involves the need for prototyping the device.

In the following algorithm module calculated device indices are compared with the limitations (1-3). If all indices (1-3) are fallen within corresponding limitations, it is concluded that the device and nanocomponent parameters are laid in operability field $\boldsymbol{O}$. If any of device parameters is out of the limitations framework, it is concluded that device and nanocomponent parametric failure is detected, in other words, the nanocomponent parameters is related to $\boldsymbol{C O}$ or $\boldsymbol{C F}$ field.

\section{Reliability-prediction technique of nanocomponent and device based on it}

The technique of reliability prediction of a nanocomponent and a device based on it includes a decision-making algorithm. The block diagram of the algorithm of the reliability prediction of a nanocomponent and a device based on it is shown in Fig. 3. The technique is based on the sequential modeling of cycles of destabilizing effects (artificial aging) on nanocomponent, the dose of which gradually accumulates in each cycle and causes to a change in the parameters of a nanocomponent and a device based on it. The intensity $w$, the dose $d$, the duration $t$ and the number of stress cycles are chosen based on operating conditions, a priori information on the sensitivity of nanocomponent parameters to destabilizing effects, the required accuracy of the predictive reliability and modeling laboriousness.

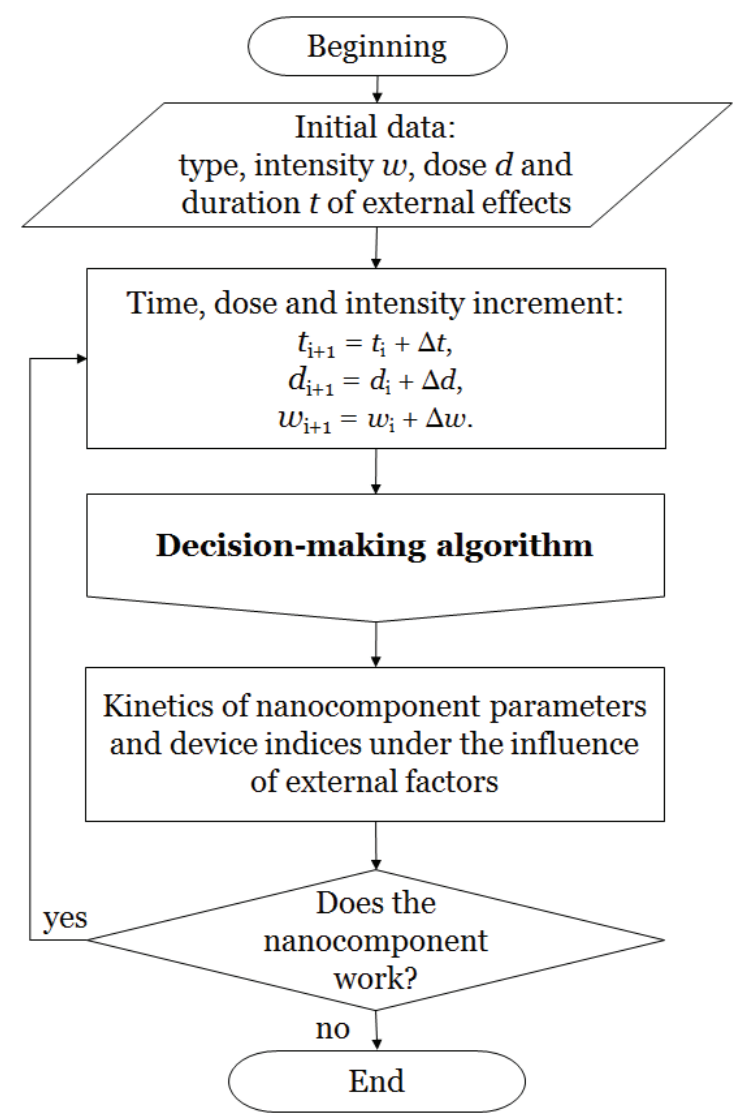

Fig. 3. The block diagram of the algorithm of the reliability prediction of a nanocomponent and a device based on it.

The technique of reliability prediction assumes conducting nanocomponent accelerated aging, during which destabilizing effects accelerate the degradation processes. The type, intensity and duration of effects are selected in such a way that the acceleration occurs only for a failure mechanism, which is typical for normal operating conditions of the device [12]. In practice for the semiconductor devices degradation rate increasing an elevated temperature is often used. The number of stress cycles is chosen at least from 4 to 5 so that the nanocomponent parameters kinetics can be estimated. The minimum intensity and duration of a stress cycle are chosen, so that the consequent change of the nanocomponent parameters were not less than by an order of magnitude larger than the error of the measuring instrument. The maximum intensity and duration of a stress cycle are limited by the risk of nanocomponent catastrophic failure. Thus, nanocomponent parameters comes to the "decision-making rules" block input after one cycle of destabilizing effects, at the output - the classification of a nanocomponent as failed, or workable.

The exit from the cycle "destabilizing effect decision-making rule" is carried out immediately after the device and nanocomponent failure at the time $t_{\mathrm{i}}$ is fixed. As a result kinetics of the nanocomponent parameters $I(w, d, t)$ and device indices under the effect of external factors are revealed. On this basis estimation of the nanocomponent time to failure under given conditions is carry out. 
The results of the RTD parameter kinetic studies under the influence of the elevated temperature are shown in [13-16].

The given algorithm makes it possible to obtain a point deterministic estimation of the time to failure of a nanocomponent and a device based on it. In addition, algorithm will allow constructing the MTBF statistical distribution and determining the necessary reliability characteristics of a production batches by adding to the algorithm technological errors of a nanocomponent and the device based on it, as well as the operational effects randomness.

\section{Conclusions}

The nanocomponent operability field is determined by the operability field of the device based on it, its parameters and application conditions. The nanocomponent time to failure is determined by its operability field and application conditions.

Consideration of the proposed technique and algorithms with respect to the mixing RTD does not limit their generality. The considered technique and algorithms can be applied to the construction of the operability field and time to failure forecast under given conditions of any nanocomponent of any device whose indices depend on the nanocomponent parameters.

The considered technique and algorithms can be used in production conditions during testing and planning of technological operations for monitoring the nanocomponent parameters.

The research work was supported by Ministry of Education and Science of the Russian Federation under state task № 16.1663.2017/4.6.

\section{References}

1. I.A. Birger, Tehnicheskaja diagnostika (Mashinostroenie, Moscow, 1978)

2. H. Czichos (ed.), Handbook of technical diagnostics: fundamentals and application to structures and systems (Springer Science \& Business Media, 2013)

3. H. Mizuta, T. Tanoue, High-speed and functional applications of resonant tunnelling diodes. In The Physics and Applications of Resonant Tunnelling Diodes (Cambridge university press, 2006)

4. S. Suzuki, M. Asada, A. Teranishi et al., Appl. Phys. Lett. 97 (24) 242102 (2010)

5. H. Kanaya et al., Applied Physics Express 5 (12) 124101 (2012).

6. T. Maekawa et al., Applied Physics Express 9 (2) 024101 (2016).

7. Y.A. Ivanov, S.A. Meshkov, I.A. Fedorenko et al, J. Commun. Technol. Electron 55 921-927 (2010)

8. Y.A. Ivanov et al., Microwave \& Telecommunication Technology (CriMiCo), 2014 24th International Crimean Conference, IEEE. 1063-1064 (2014)
9. A.G. Gudkov, Y.A. Ivanov, S.A. Meshkov et al, Biomedical Engineering 49 98-101 (2015)

10. V.Y. Sinyakin, M.O. Makeev, S.A. Meshkov, J. Phys.: Conf. Ser. 741012160 (2016)

11. A.S. Pronikov, Parametric reliability of machines (Bauman University Publishing House, Moscow, 2002)

12. O.P. Gludkin, Metody $i$ ustrojstva ispytanij RES $i$ EVS (Vysshaja shkola, Moscow, 1991)

13. M.O. Makeev, Y.A. Ivanov, S.A. Meshkov, Journal of Physics: Conference Series 584012014 (2015)

14. M.O. Makeev, S.A. Meshkov, Y.A. Ivanov, Key Engineering Materials 724 48-52 (2017)

15. M.O. Makeev, Y.A. Ivanov, S.A. Meshkov et al, 2015 5th International Workshop on Computer Science and Engineering: Information Processing and Control Engineering, WCSE 2015-IPCE. 260265 (2015)

16. M. O. Makeev, S. A. Meshkov, AIP Conference Proceedings 1858020001 (2017) 